\title{
Fuzzy quantic nuclei and conuclei with applications to fuzzy semi-quantales and $(L, M)$-quasi-fuzzy topologies
}

\author{
Kamal El-Saady*, Saad Sharqawy and Ayat A. Temraz
}

\section{${ }^{*}$ Correspondence:}

kehassan@sci.svu.edu.eg

Department of Mathematics,

Faculty of Science, South Valley

University, Qena, 83523, Egypt

\begin{abstract}
The aim of this paper is to introduce and study the notions of $M$-fuzzy quantic nuclei and conuclei on quantales. Firstly, the concept of an $M$-fuzzy quantic nuclei is introduced and some of its properties are discussed. Secondly, the concept of an M-fuzzy quantic conuclei is introduced. As an application of $M$-fuzzy quantic conuclei on quantales, a characterization of an $(L, M)$-quasi-fuzzy interior operator on a non-empty set $X$ is given and the relationship between it and an $(L, M)$-quasi-fuzzy topology is discussed. Finally, the concept of an $M$-fuzzy left (resp., right) ideal conucleus is introduced and the relationship with the concept of $M$-fuzzy left (resp., right) ideal is introduced.
\end{abstract}

Keywords: Quantale, Semi-quantale, Fuzzy semi-quantale, Quantic nucleus and conucleus

AMS subject classification: $06 \mathrm{~A} 06,54 \mathrm{~A} 10,54 \mathrm{~A} 40$

\section{Introduction}

Quantales were introduced by C. J. Mulvey in [1], with the purpose of studying the foundations of quantum mechanics and the spectrum of $C^{*}$-algebras. In 2007, Rodabaugh [2] introduced the notion of semi-quantale as a generalization of quantale and used it as an appropriate lattice-theoretic basis to formulate powerset, topological, and fuzzy topological theories. The notion of semi-quantale provides a useful tool to gather various lattice-theoretic notions, which have been extensively studied in non-commutative structures; it has a wide application, especially in studying the non-commutative lattice-valued quasi-topology [2-6].

In 2015, Demirici [7] introduced the notion of $M$-fuzzy semi-quantales as a fuzzy version of notion of Rodabaugh's semi-quantales, providing a common framework for $(L, M)$-fuzzy topological spaces of Kubiak and Šstak [8], $L$-quasi-fuzzy topological spaces of Rodabaugh [2], and $L$-fuzzy topological spaces of Höhle and S̆ostak [9].

As we all know, the quantic nuclei and the quantic conuclei play an important role in quantale theory. In this paper, we aim to introduce the notions of $M$-fuzzy quantic nuclei and conuclei on quantales and study some of their properties. Firstly, in "The (direct) product of two quantales" section, we will define and study the (direct) product of two quantales which will be used through this paper. In " $M$-fuzzy quantic nuclei" section, the concept of an $M$-fuzzy quantic ( or quantale) nuclei is introduced and

(c) The Author(s). 2019 Open Access This article is distributed under the terms of the Creative Commons Attribution 4.0 International License (http://creativecommons.org/licenses/by/4.0/), which permits unrestricted use, distribution, and reproduction in any medium, provided you give appropriate credit to the original author(s) and the source, provide a link to the Creative Commons license, and indicate if changes were made. 
a relationship between it and the notion of $M$-fuzzy semi-quantales is discussed. In " $M$-fuzzy quantic conuclei" section, the concept of an $M$-fuzzy quantic (or quantale) conuclei is introduced. As an application of $M$-fuzzy quantic conuclei on quantales, we characterize and study the notion of $(L, M)$-quasi-fuzzy interior operator on a non-empty set $X$ and discuss the relationship between it and an $(L, M)$-quasi-fuzzy topology on $X$. Finally, in " $M$-fuzzy ideal conuclei on quantales" section, the concept of an $M$-fuzzy left (resp., right) ideal conucleus is introduced and the relationship with the concept of $M$-fuzzy left (resp., right) quantale ideals is introduced.

\section{Preliminaries}

A semi-quantale $L=(L, \leq, \otimes)[2,10]$ is a complete lattice $L=(L, \leq)$ equipped with a binary operation $\otimes: L \times L \longrightarrow L$ (called a tensor product) with no additional assumptions. As convention, we denote the join, meet, top, and bottom in the complete lattice $(L, \leq)$ by $\bigvee, \bigwedge, \top_{L}$, and $\perp_{L}$, respectively. Semi-quantales include various classes of ordered algebraic structures (e.g., complete residuated lattices, unit interval $[0,1]$ equipped with $t$-norms or $t$-conorms, quantales, frames, semi-frames) playing a major role in fuzzy set theory and fuzzy logics $[11,12]$. Now, we list only some of their definitions that will be needed in the following text.

Definition 1 A semi-quantale $L=(L, \leq, \otimes)$ is called:

(1) A unital semi-quantale [2] if the groupoid $(L, \otimes)$ has an identity element $e \in L$ called the unit. If the unit e coincides with the top element $T_{L}$ of $L$, then a unital semi-quantale is called a strictly two-sided semi-quantale.

(2) A commutative semi-quantate [2] if $\otimes$ is commutative, i.e., $a \otimes b=b \otimes a$ for every $a, b \in L$.

(3) A quantale [13] if the binary operation $\otimes$ is associative and satisfies

$$
a \otimes\left(\bigvee_{i \in I} b_{i}\right)=\bigvee_{i \in I}\left(a \otimes b_{i}\right) \text { and }\left(\bigvee_{i \in I} b_{i}\right) \otimes a=\bigvee_{i \in I}\left(b_{i} \otimes a\right) \text { for all } a \in L,\{b\}_{i \in I} \subseteq L .
$$

(4) A coquantale [14] if the multiplication $\otimes$ is associative and satisfies

$$
a \otimes\left(\bigwedge_{i \in I} b_{i}\right)=\bigwedge_{i \in I}\left(a \otimes b_{i}\right) \text { and }\left(\bigwedge_{i \in I} b_{i}\right) \otimes a=\bigwedge_{i \in I}\left(b_{i} \otimes a\right) \text { for all } a \in L,\{b\}_{i \in I} \subseteq L .
$$

A semi-quantale morphism [2] $h$ from a semi-quantale $L=(L, \leq, \otimes)$ to an other semiquantale $M=(M, \leq, \odot)$ is a map $h: L \longrightarrow M$ preserving the tensor product and the arbitrary joins. If a semi-quantale morphism $h: L \longrightarrow M$ additionally preserves the top (resp., unit) element, i.e., $h\left(T_{L}\right)=\top_{M}$ (resp., $h\left(e_{L}\right)=e_{M}$ ), then it is said to be strong (resp., unital). The category SQuant comprises all semi-quantales together with semi-quantale morphisms. The non-full subcategory UnSQuant of SQuant comprises all unital semi-quantales and all unital semi-quantale morphisms [2]. Quant is the full subcategory of SQuant, which has as objects all quantales.

CoQuant is the full subcategory of SQuant, which has as objects all coquantales and as morphisms, all maps that preserve the tensor product and arbitrary meets.

Let $X$ be a non-empty set and $L \in|\mathbf{S Q u a n t}|$. An $L$-fuzzy subset (or $L$-subset) of $X$ is a mapping $A: X \longrightarrow L$. The family of all $L$-fuzzy subsets on $X$ will be denoted by $L^{X}$. The smallest element and the largest element in $L^{X}$ are denoted by $\perp$ and $I$, respectively. 
The algebraic and lattice-theoretic structures can be extended from the semi-quantale $(L, \leq, \bigvee, \otimes)$ to $L^{X}$ pointwisely:

- $A \leq B \Leftrightarrow A(x) \leq B(x)$,

- $(A \otimes B)(x)=A(x) \otimes B(x)$,

for all $x \in X$.

Obviously, $(L, \leq, \otimes)$ is again a semi-quantale with respect to the multiplication $\otimes$ and the joins of a subset $\left\{A_{i}: i \in I\right\}$ of $L^{X}$ is given by

$\left(\bigvee_{i \in I} A_{i}\right)(x)=\bigvee_{i \in I} A_{i}(x) \forall x \in X$

In the case where $L$ is unital with unit $e$, then $L^{X}$ becomes a unital semi-quantale with the unit $\underline{e}$. For an ordinary mapping $f: X \longrightarrow Y$, one can define the mappings $f_{L} \rightarrow$ $L^{X} \longrightarrow L^{Y}$ and $f_{L}^{\leftarrow}: L^{Y} \longrightarrow L^{X}$ by $f_{L}^{\rightarrow}(A)(y)=\bigvee\{A(x): x \in X, f(x)=y\}$ for every $A \in L^{X}$ and every $y \in Y, f_{L}^{\leftarrow}(B)=B \circ f$ for every $B \in L^{Y}$, respectively. For more details, we refer to $[2,15]$.

Every quantale $L$ has two residuals, which are induced by its binary operation $\otimes$ and which are defined by $a \searrow b=\bigvee\{c: a \otimes c \leq b\}$ and $b \swarrow a=\bigvee\{c: c \otimes a \leq b\}$, respectively, providing a single residuum $\rightarrow$ in case of a commutative multiplication (resulting complete residuated lattices of Denniston et al. [16]). These operations have the standard properties of poset adjunctions [17] or (order preserving) Galois connections [18], for example,

$$
a \otimes b \leq c \Leftrightarrow a \leq b \searrow c \Leftrightarrow b \leq c \swarrow a .
$$

For the convenience of the reader, the following proposition recalls some of their other properties, which will be heavily used throughout this paper.

Proposition $1[13,19]$ For $L \in \mid$ Quant $\mid$ with $a, b, c \in L$ and $B \subseteq L$, we have the following properties:

(1) $a \otimes(a \searrow b) \leq b$ and $(b \swarrow a) \otimes a \leq b$,

(2) $b \searrow(a \searrow c)=(a \otimes b) \searrow c$ and $(c \swarrow b) \swarrow a=c \swarrow(a \otimes b)$,

(3) $a \searrow(c \swarrow b)=(a \searrow c) \swarrow b$.

(4) $a \leq b$ implies $c \searrow a \leq c \searrow b$ and $b \searrow c \leq a \searrow c$.

Before going too much further, we recall that if $L=(L, \leq)$ is a poset, an order preserving function $g: L \rightarrow L$ is called a closure (resp., coclosure) operator on the poset $L=(L, \leq)$ $[13,17]$ iff it satisfies the following conditions:

(1) $a \leq g(a)$ (resp., $g(a) \leq a$ ), for all $a \in L$,

(2) $g(g(a))=g(a)$, for all $a \in L$.

Definition $2[4,13]$ Let $(L, \leq, \otimes) \in \mid$ SQuant $\mid$. A quantic nucleus (resp., conucleus) on $L$ is a closure (resp., coclosure) operator $g: L \longrightarrow L$ such that $g(a) \otimes g(b) \leq g(a \otimes b)$ ) for all $a, b \in L$.

Definition 3 [20] Let $L$ be a quantale. A non-empty subset $I \subseteq L$ is called a left (resp., right) ideal of $L$ if it satisfies the following three conditions: 
(1) $a \vee b \in I$ for all $a, b \in I$,

(2) For all $a, b \in L$, if $a \in I$ and $b \leq a$, then $b \in I$;

(3) For all $a \in L$ and $x \in I, a \otimes x \in I$ (resp., $x \otimes a \in I$ ).

A subset $I$ is an ideal if it is both a left ideal and a right ideal.

Definition 4 [7] Let $(L, \leq, \otimes),(M, \leq, \odot) \in \mid$ SQuant $\mid$.

(i) An M-fuzzy semi-quantale on $L$ is a map $\mu: L \longrightarrow M$ satisfying the following conditions: For all $a, b \in L$ and $\left\{a_{j} \mid j \in J\right\} \subseteq L$,

$(F S Q 1) \quad \mu(a) \odot \mu(b) \leq \mu(a \otimes b)$,

(FSQ2) $\bigwedge_{j \in J} \mu\left(a_{j}\right) \leq \mu\left(\bigvee_{j \in J} a_{j}\right)$.

(ii) An M-fuzzy semi-quantale $\mu$ is called strong if $\mu\left(\top_{L}\right)=\top_{M}$.

(iii) In case where $(L, \leq, \otimes)$ is a unital semi-quantale with the unit $e_{L}$, an $M$-fuzzy semi-quantale $\mu$ is called unital if $\mu\left(e_{L}\right)=\top_{M}$.

Definition 5 [7] Let $(L, \leq, \otimes),(M, \leq, \odot) \in \mid$ SQuant $\mid$, and $X$ be a non-empty set.

(i) A map $\tau: L^{X} \longrightarrow M$ is called an $(L, M)$-quasi-fuzzy topology on $X$ iff $\tau$ is an

$M$-fuzzy semi-quantale on $L^{X}$, i.e., the next conditions are satisfied for all $A, B \in L^{X}$ and $\left\{A_{j} \mid j \in J\right\} \subseteq L^{X}$ :

$\left(Q T_{1}\right) \quad \tau(A) \odot \tau(B) \leq \tau(A \otimes B)$,

$\left(Q T_{2}\right) \bigwedge_{j \in J} \tau\left(A_{j}\right) \leq \tau\left(\bigvee_{j \in J} A_{j}\right)$.

(ii) An $(L, M)$-quasi-fuzzy topology is strong iff $\tau(T)=\top_{M}$.

(iii) Let $L$ be a unital semi-quantale with unit e. An $(L, M)$-quasi-fuzzy topology is then called an $(L, M)$-fuzzy topology iff $\tau(\underline{e})=\top_{M}$.

(iv) The ordered pair $(X, \tau)$ is called an $(L, M)$-quasi-fuzzy (resp., strong ( $L, M)$-quasi-fuzzy, $(L, M)$-fuzzy) topological space if $\tau$ is an $(L, M)$-quasi-fuzzy (resp., strong $(L, M)$-quasi-fuzzy, $(L, M)$-fuzzy) topology on $X$.

\section{The (direct) product of two quantales}

It is known that the (direct) product of two ordered sets $(P, \leq)$ and $(Q, \leq)$ is the ordered set $(P \times Q, \leq)[21]$, where the order relation on the product $P \times Q$ is defined as follows:

$$
(a, b) \leq(c, d) \text { in } P \times Q \Leftrightarrow a \leq c \text { in } P \text { and } b \leq d \text { in } Q .
$$

Also, the (direct) product of two semigroups $(G, \otimes)$ and $(H, \odot)$ is a semigroup $(G \times H, *)$ [22], where the binary operation $*$ on $G \times H$ is defined as follows:

$$
(a, b) *(c, d)=(a \otimes c, b \odot d) .
$$

Furthermore, the direct product of two complete lattices is a complete lattice [23]. So, we can conclude that the direct product of any two semi-quantales is again a semi-quantale with the tensor product $*$ denoted by Eq. (3).

Lemma 1 The direct product of any two quantales is a quantale.

Proof Since the direct product of any two semi-quantales is a semi-quantale, then we only prove the distributively of $\bigvee$ over the product $*$. 
Let $Q=(Q, \leq, \bigvee, *)=Q_{1} \times Q_{2}$ where $Q_{1}=\left(Q_{1}, \leq, \bigvee, \otimes\right)$ and $Q_{2}=\left(Q_{2}, \leq, \bigvee, \odot\right)$ are quantales.

For all $a, \bigvee_{i} a_{i} \in Q_{1}, b, \bigvee_{i} b_{i} \in Q_{2}$, we have

$$
\begin{aligned}
(a, b) * \bigvee_{i}\left(a_{i}, b_{i}\right)=(a, b) *\left(\bigvee_{i} a_{i}, \bigvee_{i} b_{i}\right) & =\left(a \otimes \bigvee_{i} a_{i}, b \odot \bigvee_{i} b_{i}\right), \\
& =\left(\bigvee_{i}\left(a \otimes a_{i}\right), \bigvee_{i}\left(b \odot b_{i}\right)\right), \\
& =\bigvee_{i}\left(\left(a \otimes a_{i}\right),\left(b \odot b_{i}\right)\right) .
\end{aligned}
$$

Similarly, we can prove that $\bigvee_{i}\left(a_{i}, b_{i}\right) *(a, b)=\bigvee_{i}\left(\left(a_{i} \otimes a\right),\left(b_{i} \odot b\right)\right)$

Lemma 2 For $\left(Q_{1}, \leq, \bigvee, \otimes\right),\left(Q_{2}, \leq, \bigvee, \odot\right) \in \mid$ Quant $\mid$, let $a_{1}, b_{1} \in Q_{1}$ and $a_{2}, b_{2} \in Q_{2}$. Then

(1) $\left(a_{1}, a_{2}\right) \searrow\left(b_{1}, b_{2}\right)=\left(a_{1} \searrow b_{1}, a_{2} \searrow b_{2}\right)$,

(2) $\left(b_{1}, b_{2}\right) \swarrow\left(a_{1}, a_{2}\right)=\left(b_{1} \swarrow a_{1}, b_{2} \swarrow a_{2}\right)$.

Proof

$$
\begin{aligned}
\left(a_{1} \searrow b_{1}, a_{2} \searrow b_{2}\right) & =\left(\bigvee\left\{c_{1}: a_{1} \otimes c_{1} \leq b_{1}\right\}, \bigvee\left\{c_{2}: a_{2} \odot c_{2} \leq b_{2}\right\}\right) \\
& =\bigvee\left\{\left(c_{1}, c_{2}\right): a_{1} \otimes c_{1} \leq b_{1}, a_{2} \odot c_{2} \leq b_{2}\right\}, \\
& =\bigvee\left\{\left(c_{1}, c_{2}\right):\left(a_{1} \otimes c_{1}, a_{2} \odot c_{2}\right) \leq\left(b_{1}, b_{2}\right)\right\}, \\
& =\bigvee\left\{\left(c_{1}, c_{2}\right):\left(a_{1}, a_{2}\right) *\left(c_{1}, c_{2}\right) \leq\left(b_{1}, b_{2}\right)\right\}, \\
& =\left(a_{1}, a_{2}\right) \searrow\left(b_{1}, b_{2}\right) .
\end{aligned}
$$

The item (2) can be proved similarly.

Proposition 2 Let $\left(Q_{1}, \leq, \bigvee, \otimes\right),\left(Q_{2}, \leq, \bigvee, \odot\right) \in \mid$ Quant $\mid, a, b, c \in Q_{1}$ and $a_{1}, b_{1}, c_{1} \in$ Q2. Then

(1) $\left(a, a_{1}\right) *\left(\left(a, a_{1}\right) \searrow\left(b, b_{1}\right)\right) \leq\left(b, b_{1}\right)$,

(2) $\left(\left(b, b_{1}\right) \swarrow\left(a, a_{1}\right)\right) *\left(a, a_{1}\right) \leq\left(b, b_{1}\right)$,

(3) $\left(b, b_{1}\right) \searrow\left(\left(a, a_{1}\right) \searrow\left(c, c_{1}\right)\right)=\left(\left(a, a_{1}\right) *\left(b, b_{1}\right)\right) \searrow\left(c, c_{1}\right)$,

(4) $\left(\left(c, c_{1}\right) \swarrow\left(b, b_{1}\right)\right) \swarrow\left(a, a_{1}\right)=\left(c, c_{1}\right) \swarrow\left(\left(a, a_{1}\right) *\left(b, b_{1}\right)\right)$,

(5) $\left(a, a_{1}\right) \searrow\left(\left(c, c_{1}\right) \swarrow\left(b, b_{1}\right)\right)=\left(\left(a, a_{1}\right) \searrow\left(c, c_{1}\right)\right) \swarrow\left(b, b_{1}\right)$.

Proof (1)

$$
\begin{aligned}
\left(a, a_{1}\right) *\left(\left(a, a_{1}\right) \searrow\right. & \left.\left(b, b_{1}\right)\right)=\left(a, a_{1}\right) *\left(a \searrow b, a_{1} \searrow b_{1}\right) \\
& =\left(a \otimes(a \searrow b), a_{1} \odot\left(a_{1} \searrow b_{1}\right)\right)(\text { by Proposition } 1(1)) \\
& \leq\left(b, b_{1}\right)
\end{aligned}
$$

(2)

$$
\begin{aligned}
\left(\left(b, b_{1}\right) \swarrow\left(a, a_{1}\right)\right) *\left(a, a_{1}\right) & =\left(b \swarrow a, b_{1} \swarrow a_{1}\right) *\left(a, a_{1}\right) \\
& =\left((b \swarrow a) \otimes a,\left(b_{1} \swarrow a_{1}\right) \odot a_{1}\right)(\text { by Proposition 1(1)) } \\
& \leq\left(b, b_{1}\right) .
\end{aligned}
$$


(3)

$$
\begin{aligned}
\left(b, b_{1}\right) \searrow\left(\left(a, a_{1}\right) \searrow\left(c, c_{1}\right)\right) & =\left(b, b_{1}\right) \searrow\left(a \searrow c, a_{1} \searrow c_{1}\right) \\
& =\left(b \searrow(a \searrow c), b_{1} \searrow\left(a_{1} \searrow c_{1}\right)\right) \\
& =\left((a \otimes b) \searrow c,\left(a_{1} \odot b_{1}\right) \searrow c_{1}\right)(\text { by Proposition } 1(2)) \\
& =\left(a \otimes b, a_{1} \odot b_{1}\right) \searrow\left(c, c_{1}\right)(\text { by Lemma } 2) \\
& =\left(\left(a, a_{1}\right) *\left(b, b_{1}\right)\right) \searrow\left(c, c_{1}\right)
\end{aligned}
$$

(4)

$$
\begin{aligned}
\left(\left(c, c_{1}\right) \swarrow\left(b, b_{1}\right)\right) \swarrow\left(a, a_{1}\right) & =\left(c \swarrow b, c_{1} \swarrow b_{1}\right) \swarrow\left(a, a_{1}\right) \\
& =\left((c \swarrow b) \swarrow a,\left(c_{1} \swarrow b_{1}\right) \swarrow a_{1}\right) \\
& =\left(c \swarrow(a \otimes b), c_{1} \swarrow\left(a_{1} \odot b_{1}\right)\right) \text { (by Proposition 1(2)) } \\
& =\left(c, c_{1}\right) \swarrow\left(a \otimes b, a_{1} \odot b_{1}\right) \text { (by Lemma 2) } \\
& =\left(c, c_{1}\right) \swarrow\left(\left(a, a_{1}\right) *\left(b, b_{1}\right)\right) .
\end{aligned}
$$

(5)

$$
\begin{aligned}
\left(a, a_{1}\right) \searrow\left(\left(c, c_{1}\right) \swarrow\left(b, b_{1}\right)\right) & =\left(a, a_{1}\right) \searrow\left(c \swarrow b, c_{1} \swarrow b_{1}\right) \\
& =\left(a \searrow(c \swarrow b), a_{1} \searrow\left(c_{1} \swarrow b_{1}\right)\right) \\
& =\left((a \searrow c) \swarrow b,\left(a_{1} \searrow c_{1}\right) \swarrow b_{1}\right) \text { (by Proposition 1(3)) } \\
& =\left(a \searrow c, a_{1} \searrow c_{1}\right) \swarrow\left(b, b_{1}\right) \text { (by Lemma 2) } \\
& =\left(\left(a, a_{1}\right) \searrow\left(c, c_{1}\right)\right) \swarrow\left(b, b_{1}\right) .
\end{aligned}
$$

\section{M-fuzzy quantic nuclei}

In this section, we will introduce the concept of an $M$-fuzzy quantic nuclei as a fuzzy version of the well-known quantic nuclei. Some properties of such $M$-fuzzy quantic nuclei will be studied, and the relationship between it and the notion of $M$-fuzzy semi-quantales will be discussed.

Before we go further into this section, let us begin with introducing a fuzzy version of the known closure operator on a partially ordered set.

Definition 6 For a complete lattice $(M, \leq)$ and an ordered set $(L, \leq)$, a mapping $C: L \times$ $M \longrightarrow L$ is called an $M$-fuzzy closure operator on $L$ if it satisfies the following conditions: for all $a, b \in L$ and $\alpha, \beta \in M$,

$\left(C_{1}\right) \quad C(a, \alpha) \geq a$;

$\left(C_{2}\right) \quad C(a, \alpha) \leq C(b, \beta)$, if $a \leq b$ and $\alpha \leq \beta$;

$\left(C_{3}\right) \quad C(a, \alpha)=C(C(a, \alpha), \alpha)$.

Proposition 3 Let $(L, \leq, \otimes),(M, \leq, \odot) \in \mid$ SQuant $\mid$ and $\mu: L \longrightarrow M$ be an $M$-fuzzy semi-quantale. A mapping $C_{\mu}: L \times M \longrightarrow L$ defined by the equality.

$C_{\mu}(a, \alpha)=\bigwedge\{x \in L: x \geq a, \mu(x) \geq \alpha\} \forall a \in L, \alpha \in M$,

is an $M$-fuzzy closure operator on $L$.

Proof Let $\mu: L \longrightarrow M$ be an $M$-fuzzy semi-quantale. To prove that the map $C_{\mu}$ : $L \times M \longrightarrow L$ defined by

$C_{\mu}(a, \alpha)=\bigwedge\{x \in L: x \geq a, \mu(x) \geq \alpha\} \forall a \in L, \alpha \in M$, 
is an $M$-fuzzy closure operator on $L$, we will prove that the conditions $\left(C_{1}-C_{3}\right)$ of the above definition hold.

$\left(C_{1}\right)$ By definition of $C_{\mu}$, we have $C_{\mu}(a, \alpha)=\bigwedge\{x \in L: x \geq a, \mu(x) \geq \alpha\} \geq a$.

So, $C_{\mu}(a, \alpha) \geq a$.

$\left(C_{2}\right)$ If $a \leq b$ and $\alpha \leq \beta$, then

$$
\begin{aligned}
C_{\mu}(b, \beta) & =\bigwedge\{x \in L: x \geq b, \mu(x) \geq \beta\}, \\
& \geq \bigwedge\{x \in L: x \geq b \geq a, \mu(x) \geq \beta \geq \alpha\}, \\
& =C_{\mu}(a, \alpha) .
\end{aligned}
$$

Hence, $C_{\mu}(a, \alpha) \leq C_{\mu}(b, \beta)$.

$\left(C_{3}\right)$ Since $C_{\mu}(a, \alpha) \in L$ and

$C_{\mu}\left(C_{\mu}(a, \alpha), \alpha\right)=\bigwedge\left\{x \in L: x \geq C_{\mu}(a, \alpha), \mu(x) \geq \alpha\right\}$,

we have that $\mu(x) \geq \mu\left(C_{\mu}(a, \alpha)\right) \geq \alpha$.

Then, putting $x=C_{\mu}(a, \alpha)$, we have

$C_{\mu}\left(C_{\mu}(a, \alpha), \alpha\right)=\bigwedge C_{\mu}(a, \alpha)$ and this implies

$C_{\mu}(a, \alpha) \geq C_{\mu}\left(C_{\mu}(a, \alpha), \alpha\right)$.

Also, from $\left(C_{1}\right)$, we have that

$C_{\mu}\left(C_{\mu}(a, \alpha), \alpha\right) \geq C_{\mu}(a, \alpha)$.

Then, the equality holds.

Definition 7 Let $(L, \leq, \otimes),(M, \leq, \odot) \in \mid$ SQuant $\mid$. A mapping $C: L \times M \longrightarrow L$ is called an $M$-fuzzy quantic nucleus operator on $L$ if it is an $M$-fuzzy closure operator on $L$ and satisfies the following condition: for all $a, b \in L$ and $\alpha, \beta \in M$,

$\left(C_{4}\right) \quad C(a, \alpha) \otimes C(b, \beta) \leq C(a \otimes b, \alpha \odot \beta)$.

Proposition 4 Let $(L, \leq, \otimes) \in \mid$ CoQuant $|,(M, \leq, \odot) \in|$ SQuant $\mid$ and $\mu: L \longrightarrow M$ be an $M$-fuzzy semi-quantale. The mapping $C_{\mu}: L \times M \longrightarrow L$ defined by the equality.

$C_{\mu}(a, \alpha)=\bigwedge\{u \in L: u \geq a, \mu(u) \geq \alpha\}, \forall a \in L, \alpha \in M$

is an $M$-fuzzy quantic nucleus on $L$.

Proof We only prove the condition $C_{4}$. For $a, b \in L$ and $\alpha, \beta \in M$, we have: $C_{\mu}(a, \alpha) \otimes$ $C_{\mu}(b, \beta)=\bigwedge\{u: u \in L, u \geq a, \alpha \leq \mu(u)\} \otimes \bigwedge\{v: v \in L, v \geq b, \beta \leq \mu(v)\}$,

$$
\begin{aligned}
& =\bigwedge\{u \otimes v: u, v \in L, u \geq a, v \geq b, \alpha \leq \mu(u), \beta \leq \mu(v)\}, \\
& =\bigwedge\{u \otimes v: u, v \in L, u \geq a, v \geq b, \alpha \odot \beta \leq \mu(u) \odot \mu(v)\}, \\
& \leq \bigwedge\{u \otimes v: u, v \in L, a \otimes b \leq u \otimes v, \alpha \odot \beta \leq \mu(u \otimes v)\}, \\
& \leq \bigwedge\{w: w \in L, w \geq a \otimes b, \alpha \odot \beta \leq \mu(w)\}=C_{\mu}(a \otimes b, \alpha \odot \beta) .
\end{aligned}
$$

Then $C_{\mu}(a, \alpha) \otimes C_{\mu}(b, \beta) \leq C_{\mu}(a \otimes b, \alpha \odot \beta)$.

Proposition 5 Let $(L, \leq, \otimes),(M, \leq, \odot) \in \mid$ Quant $\mid$ and $C: L \times M \longrightarrow L$ be an $M-f u z z y$ quantic nucleus on a quantale $L$, then

$$
C(a, \alpha) \searrow C(b, \beta) \leq a \searrow C(b, \beta) \text { (resp., } C(b, \beta) \swarrow C(a, \alpha) \leq C(b, \beta) \swarrow a) .
$$

Proof Since $C(a, \alpha) \geq a$, then by Proposition 1 (4), we have $C(a, \alpha) \searrow C(b, \beta) \leq a \searrow C(b, \beta)$.

The argument for the residuum $\swarrow$ proceeds similarly.

Corollary 1 For $(L, \leq, \otimes),(M, \leq, \odot) \in \mid$ Quant $\mid$. If $C: L \times M \longrightarrow L$ be an $M-$ fuzzy quantic nucleus on a quantale $L$, then 
(1) $C(b \swarrow a, \beta \swarrow \alpha) \leq C(b, \beta) \swarrow a$,

(2) $C(a \searrow b, \alpha \searrow \beta) \leq a \searrow C(b, \beta)$,

(3) $C(b \swarrow a, \beta \swarrow \alpha) \leq C(b, \beta) \swarrow C(a, \alpha)$,

(4) $C(a \searrow b, \alpha \searrow \beta) \leq C(a, \alpha) \searrow C(b, \beta)$,

for all $a, b \in L, \alpha, \beta \in M$.

Proof (1) Since $C(b \swarrow a, \beta \swarrow \alpha) \otimes C(a, \alpha) \leq C((b \swarrow a) \otimes a,(\beta \swarrow \alpha)) \odot \alpha) \leq C(b, \beta)$ and $C: L \times M \longrightarrow L$ is an $M$-fuzzy quantic nucleus on $L$, then from Proposition 5 , we have that $C(b \swarrow a, \beta \swarrow \alpha) \leq C(b, \beta) \swarrow a$. Similarly, we can prove (2).

(3) Since $C(a, \alpha) \otimes C(b \swarrow a, \beta \swarrow \alpha)=C(a, \alpha) \otimes C[(b, \beta) \swarrow(a, \alpha)]$ $\leq C[(a, \alpha) \otimes(b, \beta) \swarrow(a, \alpha)] \leq C(b, \beta)$. Thus $C(b \swarrow a, \beta \swarrow \alpha) \leq C(b, \beta) \swarrow C(a, \alpha)$.

Similarly, we can prove (4).

Lemma 3 Let $L$ be a unital quantale. For all $a, b \in L, \alpha, \beta \in M$, a mapping $C: L \times M \longrightarrow$ $L$ with $C(a, \alpha) \searrow C(b, \beta) \leq a \searrow C(b, \beta)$ and $C(b, \beta) \swarrow C(a, \alpha) \leq C(b, \beta) \swarrow a$ is an $M$-fuzzy quantic nucleus on $L$.

Proof For all $a, b \in L, \alpha, \beta \in M$, suppose that

$C(a, \alpha) \searrow C(b, \beta) \leq a \searrow C(b, \beta)$ and $C(b, \beta) \swarrow C(a, \alpha) \leq C(b, \beta) \swarrow a$.

By the unital assumption $e_{L}$, we have that:

(1) $e_{L} \otimes C(a, \alpha) \leq C(a, \alpha) \Leftrightarrow e_{L} \leq C(a, \alpha) \swarrow C(a, \alpha) \leq C(a, \alpha) \swarrow a$

$$
\Leftrightarrow e_{L} \otimes a \leq C(a, \alpha)
$$

$$
\Leftrightarrow a \leq C(a, \alpha) \text {. }
$$

(2) If $a \leq b$ and $\alpha \leq \beta$, then

$$
\begin{aligned}
e_{L} \otimes a \leq b \leq C(b, \beta) & \Leftrightarrow e_{L} \leq C(b, \beta) \swarrow a \\
& \Leftrightarrow e_{L} \leq C(b, \beta) \swarrow C(a, \alpha) \\
& \Leftrightarrow e_{L} \otimes C(a, \alpha) \leq C(b, \beta) \\
& \Leftrightarrow C(a, \alpha) \leq C(b, \beta) .
\end{aligned}
$$

(3) Since $a \leq C(a, \alpha)$, from (2), we have $C(a, \alpha) \leq C(C(a, \alpha), \alpha)$.

On the other hand, $e_{L} \otimes C(a, \alpha) \leq C(a, \alpha) \Leftrightarrow e_{L} \leq C(a, \alpha) \swarrow C(a, \alpha)$

$$
\begin{aligned}
& \Leftrightarrow e_{L} \leq C(a, \alpha) \swarrow C(C(a, \alpha), \alpha) \\
& \Leftrightarrow e_{L} \otimes C(C(a, \alpha), \alpha) \leq C(a, \alpha) \\
& \Leftrightarrow C(C(a, \alpha), \alpha) \leq C(a, \alpha), \\
& \Leftrightarrow C(C(a, \alpha), \alpha)=C(a, \alpha) .
\end{aligned}
$$

(4) From the items (1)-(2), we have, for all $a, b \in L, \alpha, \beta \in M$

$a \otimes b \leq C(a \otimes b, \alpha \odot \beta) \Leftrightarrow a \leq C(a \otimes b, \alpha \odot \beta) \swarrow b$

$$
\begin{aligned}
& \Leftrightarrow a \leq C(a \otimes b, \alpha \odot \beta) \swarrow C(b, \beta) \\
& \Leftrightarrow a \otimes C(b, \beta) \leq C(a \otimes b, \alpha \odot \beta) \\
& \Leftrightarrow C(b, \beta) \leq a \searrow C(a \otimes b, \alpha \odot \beta) \\
& \Leftrightarrow C(b, \beta) \leq C(a, \alpha) \searrow C(a \otimes b, \alpha \odot \beta) \\
& \Leftrightarrow C(a, \alpha) \otimes C(b, \beta) \leq C(a \otimes b, \alpha \odot \beta) .
\end{aligned}
$$

Thus, $C$ is a an $M$-fuzzy quantic nucleus. The right unital case follows similarly. 


\section{M-fuzzy quantic conuclei}

In this section, we will introduce and study the concept of an $M$-fuzzy quantic conuclei on a quantale $L=(L, \leq, \otimes)$. A relationship between $M$-fuzzy quantic conuclei and $M$-fuzzy semi-quantales will be discussed. Also, we characterize and study the notion of $(L, M)$ quasi-fuzzy interior operator, as an application of $M$-fuzzy quantic conuclei on quantales, and discuss the relationship between such an $(L, M)$-quasi-fuzzy interior operator and an $(L, M)$-quasi-fuzzy topology on a non-empty set $X$.

Definition 8 Let $(L, \leq)$ and $(M, \leq)$ be posets. A mapping $\kappa: L \times M \longrightarrow L$ is called an $M$-fuzzy coclosure operator on $L$ if, for all $a, b \in L$ and $\alpha, \beta \in M$, it satisfies the following conditions:

$\left(\kappa_{1}\right) \quad \kappa(a, \alpha) \leq \kappa(b, \beta)$ whenever $a \leq b, \beta \leq \alpha$.

$\left(\kappa_{2}\right) \quad \kappa(a, \alpha) \leq a$.

$\left(\kappa_{3}\right) \kappa(a, \alpha)=\kappa(\kappa(a, \alpha), \alpha)$.

Definition 9 An $M$-fuzzy coclosure operator $\kappa: L \times M \longrightarrow L$ is said to be:

(1) Strong if $\kappa\left(T_{L}, \alpha\right)=T_{L}$.

(2) Unital if $(L, \leq, \otimes)$ is unital and $\kappa\left(e_{L}, \alpha\right)=\top_{L}$.

Proposition 6 Let $(L, \leq, \otimes),(M, \leq, \odot) \in \mid$ SQuant $\mid$ and $\mu: L \longrightarrow M$ be an $M$-fuzzy (resp., a strong M-fuzzy) semi-quantale. The mapping $\kappa_{\mu}: L \times M \longrightarrow L$ defined by the equality.

$\kappa_{\mu}(a, \alpha)=\bigvee\{x \in L: x \leq a, \mu(x) \geq \alpha\} \forall a \in L$ and $\alpha \in M$;

is an $M$-fuzzy (resp., a strong $M$-fuzzy) coclosure operator on $L$.

Proof Let $\mu: L \longrightarrow M$ be an $M$-fuzzy semi-quantale. To prove that the map $\kappa_{\mu}$ : $L \times M \longrightarrow L$ defined by

$\kappa_{\mu}(a, \alpha)=\bigvee\{x \in L: x \leq a, \mu(x) \geq \alpha\} \forall a \in L$ and $\alpha \in M$

is an $M$-fuzzy coclosure operator on $L$, we will prove the conditions $\left(\kappa_{1}-\kappa_{3}\right)$ of the above definition.

( $\left.\kappa_{1}\right)$ For $a, b \in L$ and $\alpha, \beta \in M$ with $a \leq b, \beta \leq \alpha$, we have

$$
\begin{aligned}
\kappa_{\mu}(a, \alpha) & =\bigvee\{x \in L: x \leq a, \mu(x) \geq \alpha\}, \\
& \leq \bigvee\{x \in L: x \leq b, \mu(x) \geq \beta\}, \\
& =\kappa_{\mu}(b, \beta) .
\end{aligned}
$$

So, $\kappa_{\mu}(a, \alpha) \leq \kappa_{\mu}(b, \beta)$.

$\left(\kappa_{2}\right)$ By definition of $\kappa_{\mu}$, we have

$\kappa_{\mu}(a, \alpha)=\bigvee\{x \in L: x \leq a, \mu(x) \geq \alpha\} \leq a$.

Then, $\kappa_{\mu}(a, \alpha) \leq a$.

( $\left.\kappa_{3}\right)$ Since $\kappa_{\mu}(a, \alpha) \in L$ and $\kappa_{\mu}\left(\kappa_{\mu}(a, \alpha), \alpha\right)=\bigvee\left\{x \in L: x \leq \kappa_{\mu}(a, \alpha), \mu(x) \geq \alpha\right\}$, we have that $\mu\left(\kappa_{\mu}(a, \alpha)\right) \geq \mu(x) \geq \alpha$.

Then, putting $x=\kappa_{\mu}(a, \alpha)$, we have $\kappa_{\mu}\left(\kappa_{\mu}(a, \alpha), \alpha\right)=\bigvee \kappa_{\mu}(a, \alpha)$ and this implies $\kappa_{\mu}(a, \alpha) \leq \kappa_{\mu}\left(\kappa_{\mu}(a, \alpha), \alpha\right)$.

Also, from $\left(\kappa_{2}\right)$, we have that

$\kappa_{\mu}\left(\kappa_{\mu}(a, \alpha), \alpha\right) \leq \kappa_{\mu}(a, \alpha)$.

Then, the equality holds. 
If $\mu: L \longrightarrow M$ be a strong $M$-fuzzy semi-quantale, then it is clear that

$\kappa_{\mu}\left(\top_{L}, \alpha\right)=\bigvee\left\{x \in L, x=\top_{L}, \mu(x) \geq \alpha\right\}=\top_{L}$,

which means that $\kappa_{\mu}$ is a strong $M$-fuzzy coclosure operator on $L$.

Definition 10 Let $(L, \leq, \otimes),(M, \leq, \odot) \in \mid$ SQuant|. A mapping $\kappa: L \times M \longrightarrow L$ is called an $M$-fuzzy quantic conucleus on $L$ if it is an $M$-fuzzy coclosure operator on $L$ and satisfies the following conditions: for all $a, b \in L$ and $\alpha, \beta \in M$,

$\left(\kappa_{4}\right) \kappa(a, \alpha) \otimes \kappa(b, \beta) \leq \kappa(a \otimes b, \alpha \odot \beta)$.

Proposition 7 Let $(L, \leq, \otimes) \in \mid$ Quant $|,(M, \leq, \odot) \in|$ SQuant $\mid$ and $\mu: L \longrightarrow M$ be an $M$-fuzzy semi-quantale on $L$. The mapping $\kappa_{\mu}: L \times M \longrightarrow L$ defined by the equality.

$\kappa_{\mu}(a, \alpha)=\bigvee\{x \in L: x \leq a, \mu(x) \geq \alpha\} \forall a \in L, \alpha \in M$,

is an $M$-fuzzy quantic conucleus on $L$.

Proof We only prove the condition $\kappa_{4}$ :

$$
\begin{aligned}
\kappa_{\mu}(a, \alpha) \otimes \kappa_{\mu}(b, \beta) & =\bigvee\{x: x \in L, x \leq a, \alpha \leq \mu(x)\} \otimes \bigvee\{y: y \in L, y \leq b, \beta \leq \mu(y)\} \\
& =\bigvee\{x \otimes y: x, y \in L, x \leq a, y \leq b, \alpha \leq \mu(x), \beta \leq \mu(y)\}, \\
& \leq \bigvee\{z: z \in L, z \leq a \otimes b, \alpha \odot \beta \leq \mu(z)\}=\kappa_{\mu}(a \otimes b, \alpha \odot \beta) .
\end{aligned}
$$

Then , $\kappa_{\mu}(a, \alpha) \otimes \kappa_{\mu}(b, \beta) \leq \kappa_{\mu}(a \otimes b, \alpha \odot \beta)$.

Remark 1 If $L \in \mid$ UnSQuant $\mid$ and $\kappa_{\mu}\left(e_{L}, \alpha\right)=\bigvee\left\{x \in L, x=e_{L}, \mu(x) \geq \alpha\right\}=T_{L}$, then $\kappa_{\mu}$ is a unital M-fuzzy quantic conucleus on $L$.

Proposition 8 For $(L, \leq, \otimes),(M, \leq, \odot) \in \mid$ Quant| and given an $M$-fuzzy (resp., a strong $M$-fuzzy) quantic conucleus $\kappa: L \times M \longrightarrow L$, then an $M$-fuzzy set $\mu_{\kappa}: L \longrightarrow M$, which defined by

$\mu_{\kappa}(a)=\bigvee\{\alpha \in M: \kappa(a, \alpha) \geq a, a \in L\}$,

is an $M$-fuzzy (resp., a strong $M$-fuzzy) semi-quantale on $L$.

Proof Let $\kappa: L \times M \longrightarrow L$ be an $M$-fuzzy quantic conucleus on $L$. We need to show that $\mu_{\kappa}$ is an $M$-fuzzy semi-quantale. To this end

(1) For a family of $\left\{a_{i}: i \in I\right\} \subseteq L$, we have

$$
\begin{aligned}
\mu_{\kappa}\left(\bigvee_{i} a_{i}\right) & =\bigvee\left\{\alpha \in M: \kappa\left(\bigvee_{i \in I} a_{i}, \alpha\right) \geq \bigvee_{i \in I} a_{i}\right\}, \\
& =\bigvee\left\{\alpha \in M: \bigvee_{i \in I} \kappa\left(a_{i}, \alpha\right) \geq \bigvee_{i \in I} a_{i}\right\}, \\
& \geq \bigwedge_{i \in I} \bigvee\left\{\alpha \in M: \kappa\left(a_{i}, \alpha\right) \geq a_{i}\right\}=\bigwedge_{i \in I} \mu_{\kappa}\left(a_{i}\right) .
\end{aligned}
$$

Then, $\mu_{\kappa}\left(\bigvee_{i} a_{i}\right) \geq \bigwedge_{i \in I} \mu_{\kappa}\left(a_{i}\right)$.

(2) For $a, b \in L$ and $\alpha, \beta \in M$.

$$
\begin{aligned}
\mu_{\kappa}(a) \odot \mu_{\kappa}(b) & =\bigvee\{\alpha \in M: \kappa(a, \alpha) \geq a\} \odot \bigvee\{\beta \in M: \kappa(b, \beta) \geq b\}, \\
& =\bigvee\{\alpha \odot \beta: \kappa(a, \alpha) \geq a, \kappa(b, \beta) \geq b\}, \\
& =\bigvee\{\alpha \odot \beta: \kappa(a, \alpha) \otimes \kappa(b, \beta) \geq a \otimes b\}, \\
& =\bigvee\{\alpha \odot \beta: \kappa(a \otimes b, \alpha \odot \beta) \geq \kappa(a, \alpha) \otimes \kappa(b, \beta) \geq a \otimes b\}, \\
& \leq \bigvee\{\gamma: \kappa(a \otimes b, \gamma) \geq a \otimes b\}, \\
& =\mu_{\kappa}(a \otimes b) .
\end{aligned}
$$

Then, $\mu_{\kappa}(a) \odot \mu_{\kappa}(b) \leq \mu_{\kappa}(a \otimes b)$.

In the case of a strong $M$-fuzzy quantic conucleus, i.e., $\kappa\left(T_{L}, \alpha\right)=\top_{L}$, we have that $\mu_{\kappa}\left(T_{L}\right)=T_{M}$ and this completes the proof. 
The following proposition lists some of the basic properties of the residuals $\searrow$ and $\swarrow$ on an $M$-fuzzy quantic conucleus $\kappa: L \times M \longrightarrow L$.

Proposition 9 For $(L, \leq, \otimes),(M, \leq, \odot) \in \mid$ Quant $\mid$, let $\kappa: L \times M \longrightarrow L$ be an $M$-fuzzy quantic conucleus on $L$. Then for all $a, b, c \in L, \alpha, \beta \in M$, the following hold:

(i) $\kappa(a, \alpha) \otimes \kappa(a \searrow b, \alpha \searrow \beta) \leq \kappa(b, \beta)$,

(ii) $\kappa(b \swarrow a, \beta \swarrow \alpha) \otimes \kappa(a, \alpha) \leq \kappa(b, \beta)$.

Proof (1) By Proposition 1 (1), we have

$$
\begin{aligned}
\kappa(a, \alpha) \otimes \kappa(a \searrow b, \alpha \searrow \beta) & =\kappa(a, \alpha) \otimes \kappa[(a, \alpha) \searrow(b, \beta)] \\
& \leq \kappa[(a, \alpha) \otimes(a, \alpha) \searrow(b, \beta)] \text { (by Proposition 2(1)) } \\
& \leq \kappa(b, \beta) .
\end{aligned}
$$

(2) By Proposition 1 (1), we have

$$
\begin{aligned}
\kappa(b \swarrow a, \beta \swarrow \alpha) \otimes \kappa(a, \alpha) & =\kappa[(b, \beta) \swarrow(a, \alpha)] \otimes \kappa(a, \alpha) \\
& \leq \kappa[(b, \beta) \swarrow(a, \alpha) \otimes(a, \alpha)] \text { (by Proposition 2(2)); } \\
& \leq \kappa(b, \beta) .
\end{aligned}
$$

We conclude this section by given the notion of $(L, M)$-quasi-fuzzy interior operator as an example of an $M$-fuzzy quantic conucleus on the power set quantale $L^{X}$ and as a generalized form of an $L$-interior operator of [9]. Also, we study the relationship between $(L, M)$-quasi-fuzzy interior operator and $(L, M)$-quasi-fuzzy topology on a non-empty set $X$.

Definition 11 For $(L, \leq, \otimes),(M, \leq, \odot) \in \mid$ SQuant $\mid$ and a non-empty set $X$, the mapping $\mathcal{I}: L^{X} \times M \longrightarrow L^{X}$ is called:

(i) An $(L, M)$-quasi-fuzzy interior operator on $X$ iff $\mathcal{I}$ satisfies the following conditions: For all $A, B \in L^{X}, \alpha, \beta \in M$;

$\left(\mathcal{I}_{1}\right) \quad \mathcal{I}(A, \alpha) \leq \mathcal{I}(B, \beta)$ whenever $A \leq B, \beta \leq \alpha$.

$\left(\mathcal{I}_{2}\right) \quad \mathcal{I}(A, \alpha) \leq A$.

$\left(\mathcal{I}_{3}\right) \quad \mathcal{I}(A, \alpha) \leq \mathcal{I}(\mathcal{I}(A, \alpha), \alpha)$

$\left(\mathcal{I}_{4}\right) \quad \mathcal{I}(A, \alpha) \otimes \mathcal{I}(B, \beta) \leq \mathcal{I}(A \otimes B, \alpha \odot \beta)$.

(ii) A strong $(L, M)$-quasi-fuzzy interior operator if it satisfies the following condition:

( $\left.\mathcal{I}_{5}\right) \quad \mathcal{I}(\underline{T}, \alpha)=\underline{T}$.

(iii) An ( $L, M)$-fuzzy interior operator if $L \in \mid$ UnSQuant $\mid$ with unit e and the following condition is satisfied:

$\left(\mathcal{I}_{6}\right) \quad \mathcal{I}(\underline{e}, \alpha)=\underline{T}$.

Proposition 10 For $(L, \leq, \otimes),(M, \leq, \odot) \in \mid$ Quant $\mid$, a non-empty set $X$, and an $(L, M)$ quasi-fuzzy topology $\tau: L^{X} \longrightarrow M$, the mapping $\mathcal{I}_{\tau}: L^{X} \times M \longrightarrow L^{X}$ defined by the equality.

$\mathcal{I}_{\tau}(A, \alpha)=\bigvee\left\{u \in L^{X}: u \leq A, \tau(u) \geq \alpha\right\}, \forall A \in L^{X}, \alpha \in M$,

is an $(L, M)$-quasi-fuzzy interior operator on $X$.

Proof Let $\tau: L^{X} \longrightarrow M$ be an $(L, M)$-quasi-fuzzy topology on $X$. To prove that the map $\mathcal{I}_{\tau}: L^{X} \times M \longrightarrow L^{X}$ defined by 
$\mathcal{I}_{\tau}(A, \alpha)=\bigvee\left\{u \in L^{X}: u \leq A, \tau(u) \geq \alpha\right\}, \forall A \in L^{X}, \alpha \in M$,

is an $(L, M)$-quasi-fuzzy interior operator on $X$, we will prove that the conditions $\left(\mathcal{I}_{1}-\right.$

$\mathcal{I}_{4}$ ) of the above definition hold.

(1) For $A, B \in L^{X}$ and $\alpha, \beta \in M$ with $A \leq B, \beta \leq \alpha$, we have

$$
\begin{aligned}
\mathcal{I}_{\tau}(A, \alpha) & =\bigvee\left\{u \in L^{X}: u \leq A, \tau(u) \geq \alpha\right\}, \\
& \leq \bigvee\left\{u \in L^{X}: u \leq B, \tau(u) \geq \beta\right\}, \\
& =\mathcal{I}_{\tau}(B, \beta) .
\end{aligned}
$$

(2) By definition of $\mathcal{I}_{\tau}$, we have $\mathcal{I}_{\tau}(A, \alpha)=\bigvee\left\{u \in L^{X}: u \leq A, \tau(u) \geq \alpha\right\} \leq A$.

(3) Since $\mathcal{I}_{\tau}(A, \alpha) \in L^{X}$ and $\mathcal{I}_{\tau}\left(\mathcal{I}_{\tau}(A, \alpha), \alpha\right)=\bigvee\left\{u \in L^{X}: u \leq \mathcal{I}_{\tau}(A, \alpha), \tau(u) \geq \alpha\right\}$, then we have that $\tau\left(\mathcal{I}_{\tau}(A, \alpha)\right) \geq \tau(u) \geq \alpha$.

Putting $u=\mathcal{I}_{\tau}(A, \alpha)$, we have $\mathcal{I}_{\tau}\left(\mathcal{I}_{\tau}(A, \alpha), \alpha\right)=\bigvee \mathcal{I}_{\tau}(A, \alpha)$ and this implies

$\mathcal{I}_{\tau}(A, \alpha) \leq \mathcal{I}_{\tau}\left(\mathcal{I}_{\tau}(A, \alpha), \alpha\right)$.

Also, from (2), we have that

$\mathcal{I}_{\tau}\left(\mathcal{I}_{\tau}(A, \alpha), \alpha\right) \leq \mathcal{I}_{\tau}(A, \alpha)$.

Then, the equality holds.

(4) $\mathcal{I}_{\tau}(A, \alpha) \otimes \mathcal{I}_{\tau}(B, \beta)=\bigvee\left\{u: u \in L^{X}, u \leq A, \alpha \leq \tau(u)\right\} \otimes \bigvee\left\{v: v \in L^{X}, v \leq B, \beta \leq\right.$ $\tau(v)\}$,

$$
\begin{aligned}
& =\bigvee\left\{u \otimes v: u, v \in L^{X}, u \leq A, v \leq B, \alpha \leq \tau(u), \beta \leq \tau(v)\right\} \\
& \leq \bigvee\left\{w: w \in L^{X}, w \leq A \otimes B, \alpha \odot \beta \leq \tau(w)\right\}=\mathcal{I}_{\tau}(A \otimes B, \alpha \odot \beta) .
\end{aligned}
$$

That is, $\mathcal{I}_{\tau}(A, \alpha) \otimes \mathcal{I}_{\tau}(B, \beta) \leq \mathcal{I}_{\tau}(A \otimes B, \alpha \odot \beta)$.

As consequences of the above proposition, we have the following result:

Corollary 2 Let $(L, \leq, \otimes),(M, \leq, \odot) \in \mid$ Quant $\mid$ and $X$ be a non-empty set.

(1) For a strong $(L, M)$-quasi-fuzzy topology $\tau: L^{X} \longrightarrow M$, the mapping $\mathcal{I}_{\tau}: L^{X} \times M \longrightarrow L^{X}$ is a strong $(L, M)$-quasi-fuzzy interior operator on $X$.

(2) For $L \in \mid$ UnQuant $\mid$ and an $(L, M)$-fuzzy topology $\tau: L^{X} \longrightarrow M$, the mapping $\mathcal{I}_{\tau}: L^{X} \times M \longrightarrow L^{X}$ is an $(L, M)$-fuzzy interior operator on $X$.

Proposition 11 For $(L, \leq, \otimes),(M, \leq, \odot) \in \mid$ Quant $\mid$ and an ( $L, M)$-quasi-fuzzy (resp., strong $(L, M)$-quasi-fuzzy, $(L, M)$-fuzzy) interior operator $\mathcal{I}: L^{X} \times M \longrightarrow L^{X}$, the mapping $\tau_{\mathcal{I}}: L^{X} \longrightarrow M$ defined by $\tau_{\mathcal{I}}(A)=\bigvee\{\alpha \in M: \mathcal{I}(A, \alpha) \geq A\}, A \in L^{X}$,

is an $(L, M)$-quasi-fuzzy (resp., strong $(L, M)$-quasi-fuzzy, $(L, M)$-fuzzy) topology on $X$.

Proof We prove only the case of $(L, M)$-quasi-fuzzy interior operator, and the other cases can be proved similarly. Let $\mathcal{I}: L^{X} \times M \longrightarrow L^{X}$ be an $(L, M)$-quasi-fuzzy interior operator on $X$. Define the mapping $\tau_{\mathcal{I}}: L^{X} \longrightarrow M$ by $\tau_{\mathcal{I}}(A)=\bigvee\{\alpha \in M: \mathcal{I}(A, \alpha) \geq A\}, A \in L^{X}$.

We need to show that $\tau_{\mathcal{I}}$ is an $(L, M)$-quasi-fuzzy topology on $X$. To this end

(1) For a family of $\left\{A_{i}: i \in I\right\} \subseteq L^{X}$, we have

$$
\begin{aligned}
\tau_{\mathcal{I}}\left(\bigvee_{i} A_{i}\right) & =\bigvee\left\{\alpha \in M: \mathcal{I}\left(\bigvee_{i \in I} A_{i}, \alpha\right) \geq \bigvee_{i \in I} A_{i}\right\} \\
& =\bigvee\left\{\alpha \in M: \bigvee_{i \in I} \mathcal{I}\left(A_{i}, \alpha\right) \geq \bigvee_{i \in I} A_{i}\right\}, \\
& \geq \bigwedge_{i \in I} \bigvee\left\{\alpha \in M: \mathcal{I}\left(A_{i}, \alpha\right) \geq A_{i}\right\}=\bigwedge_{i \in I} \tau_{\mathcal{I}}\left(A_{i}\right)
\end{aligned}
$$

Then, $\tau_{\mathcal{I}}\left(\bigvee_{i} A_{i}\right) \geq \bigwedge_{i \in I} \tau_{\mathcal{I}}\left(A_{i}\right)$. 
(2) For $A, B \in L^{X}$ and $\alpha, \beta \in M$,

$$
\begin{aligned}
\tau_{\mathcal{I}}(A) \odot \tau_{\mathcal{I}}(B) & =\bigvee\{\alpha \in M: \mathcal{I}(A, \alpha) \geq A\} \odot \bigvee\{\beta \in M: \mathcal{I}(B, \beta) \geq B\}, \\
& =\bigvee\{\alpha \odot \beta: \mathcal{I}(A, \alpha) \geq A, \mathcal{I}(B, \beta) \geq B\}, \\
& =\bigvee\{\alpha \odot \beta: \mathcal{I}(A \otimes B, \alpha \odot \beta) \geq A \otimes B\}, \\
& \leq \bigvee\{\gamma: \mathcal{I}(A \otimes B, \gamma) \geq A \otimes B\}, \\
& =\tau_{\mathcal{I}}(A \otimes B) .
\end{aligned}
$$

Then, $\tau_{\mathcal{I}}(A) \odot \tau_{\mathcal{I}}(B) \leq \tau_{\mathcal{I}}(A \otimes B)$.

Remark 2 The correspondences $\tau \longmapsto \mathcal{I}_{\tau}$ and $\mathcal{I} \longmapsto \tau_{\mathcal{I}}$ obtained in Propositions 10 and 11 are the generalizations of the correspondences between L-fuzzy interior operators and L-fuzzy topological spaces in [9].

\section{M-fuzzy ideal conuclei on quantales}

In this section, we define $M$-fuzzy left (resp., right) ideals of quantales and discuss some of their properties. Also, the concept of an $M$-fuzzy left (resp., right) ideal conucleus is introduced and the relationship with the concept of $M$-fuzzy left (resp., right) ideals on a quantale is introduced.

Definition 12 Let $(L, \leq, \otimes),(M, \leq, \odot) \in|\mathbf{S Q u a n t}|$. An $M$-fuzzy left (resp, right) ideal on a quantale $L$ is a map $\mu: L \longrightarrow M$ satisfying the following conditions: for all $a, b \in X$

(I $\left.I_{1}\right)$ If $a \leq b$, then $\mu(a) \geq \mu(b)$.

(I $\left.I_{2}\right) \mu(a \vee b) \geq \mu(a) \wedge \mu(b)$.

(I $\left.I_{3}\right) \mu(a \otimes b) \geq \mu(b)($ resp., $\mu(a))$.

A map $\mu: L \longrightarrow M$, which is both $M$-fuzzy left and right ideal, is called an M-fuzzy ideal.

Example 1 Let $L=\{\perp, a, b, c, d, \top\}$ be a set ordered by $\perp \leq c \leq b \leq \top, \perp \leq d \leq a \leq \top$, and $d \leq b$ and equipped with associative binary operations:

\begin{tabular}{l|llllll}
$\otimes$ & $\perp$ & $a$ & $b$ & $c$ & $d$ & $\top$ \\
\hline$\perp$ & $\perp$ & $\perp$ & $\perp$ & $\perp$ & $\perp$ & $\perp$ \\
$a$ & $\perp$ & $a$ & $d$ & $\perp$ & $d$ & $a$ \\
$b$ & $\perp$ & $d$ & $c$ & $c$ & $\perp$ & $b$ \\
$c$ & $\perp$ & $\perp$ & $c$ & $c$ & $\perp$ & $c$ \\
$d$ & $\perp$ & $d$ & $\perp$ & $\perp$ & $\perp$ & $d$ \\
$\top$ & $\perp$ & $a$ & $b$ & $c$ & $d$ & $T$ \\
\hline
\end{tabular}

Then, we can easily see that $(L, \leq, \otimes)$ is a quantale. A mapping $\mu: L \longrightarrow\{0,1\}$ defined by

$$
\mu(x)=\left\{\begin{array}{cc}
1 & x \leq b \\
0 & \text { otherwise }
\end{array}\right.
$$

is an $M$ - fuzzy left(resp., right) ideal of the quantale $(L, \leq, \otimes)$.

Proposition 12 Let $\left\{\mu_{j}\right\}_{j \in J}$ be a family of M-fuzzy left (resp., right) ideals of a quantale $L$. Then, $\bigwedge_{j \in J} \mu_{j}$ is also an $M$-fuzzy left (resp., right) ideal of $L$. 
Proof Suppose that $\left\{\mu_{j}\right\}_{j \in J}$ be a family of $M$-fuzzy left (resp., right) ideals of $L$. Statement $\left(I_{1}\right)$ is clear. To prove $\left(I_{2}\right)$ notice that since every $\mu_{j}$ is an $M$-fuzzy left (resp., right) ideal of $L$, we have

$$
\begin{aligned}
\left(\bigwedge_{j \in J} \mu_{j}\right)(a \vee b) & =\bigwedge_{j \in J} \mu_{j}(a \vee b), \\
& \geq \bigwedge_{j \in J}\left(\mu_{j}(a) \wedge \mu_{j}(b)\right), \\
& \geq \bigwedge_{j \in J}\left(\mu_{j}(a)\right) \wedge \bigwedge_{j \in J}\left(\mu_{j}(b)\right), \\
& =\left(\bigwedge_{j \in J} \mu_{j}\right)(a) \wedge\left(\bigwedge_{j \in J} \mu_{j}\right)(b) .
\end{aligned}
$$

We prove property $\left(I_{3}\right)$ as follows:

$$
\begin{aligned}
\left(\bigwedge_{j \in J} \mu_{j}\right)(a \otimes b) & =\bigwedge_{j \in J} \mu_{j}(a \otimes b) \\
& \geq \bigwedge_{j \in J}\left(\mu_{j}(b)\right)\left(\text { resp. }, \bigwedge_{j \in J}\left(\mu_{j}(a)\right)\right) \\
& \geq\left(\bigwedge_{j \in J} \mu_{j}\right)(b)\left(\text { resp. },\left(\bigwedge_{j \in J} \mu_{j}\right)(a)\right) .
\end{aligned}
$$

Therefore, $\bigwedge_{j \in J} \mu_{j}$ is an $M$-fuzzy left (resp., right) ideal of $L$.

Proposition 13 An onto quantale homomorphic preimage of an M-fuzzy left (resp., right) ideal is an M-fuzzy left (resp., right) ideal.

Proof Let $f: L_{1} \longrightarrow L_{2}$ be an onto homomorphism. Let $\rho$ be an $M$-fuzzy left ideal and let $\mu$ be the preimage of $\rho$ under $f$, i.e., $\mu=f_{M}^{\overleftarrow{ }}(\rho)$. Property $\left(I_{1}\right)$ is clear. For any $a, b \in L_{1}$,

$$
\begin{aligned}
\mu(a \vee b) & =\rho(f(a \vee b)), \\
& =\rho(f(a) \vee f(b)), \\
& \geq \rho(f(a)) \wedge \rho(f(b)), \\
& =\mu(a) \wedge \mu(b) .
\end{aligned}
$$

and $\mu(a \otimes b)=\rho(f(a \otimes b))$,

$$
\begin{aligned}
& =\rho(f(a) \otimes f(b)), \\
& \geq \rho(f(b))=\mu(b) .
\end{aligned}
$$

This shows that $\mu$ is an $M$-fuzzy left ideal of $L_{1}$. The other case is similar.

Now, we are in a position to introduce and study the notion of $M$-fuzzy left (resp., right) ideal conucleus on quantales, and study the relationship with $M$-fuzzy left (resp., right) ideals.

Definition 13 For $(L, \leq, \otimes),(M, \leq, \odot) \in \mid$ Quant $\mid$ and all $a, b \in L, \alpha, \beta \in M$, an $M$ fuzzy coclosure operator $\kappa: L \times M \longrightarrow L$ is said to be:

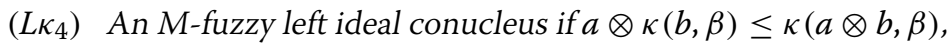

$\left(R \kappa_{4}\right)$ An M-fuzzy right ideal conucleus if $\kappa(a, \alpha) \otimes b \leq \kappa(a \otimes b, \alpha)$.

Proposition 14 Let $(L, \leq, \otimes),(M, \leq, \odot) \in \mid$ Quant $\mid$. If $\mu: L \longrightarrow M$ is an $M$-fuzzy left (resp., right) ideal on $L$, the mapping $\kappa_{\mu}: L \times M \longrightarrow L$ defined by the equality $\kappa_{\mu}(a, \alpha)=\bigvee\{x \in L: x \leq a, \mu(x) \geq \alpha\}, \forall a \in L, \alpha \in M$, is an $M$-fuzzy left (resp., right) ideal conucleus on $L$.

Proof Let $\mu: L \longrightarrow M$ be an $M$-fuzzy left ideal of $L$ and let $\kappa_{\mu}: L \times M \longrightarrow L$ be a mapping defined by

$\kappa_{\mu}(a, \alpha)=\bigvee\{x \in L: x \leq a, \mu(x) \geq \alpha\} \forall a \in L, \alpha \in M$.

We need to show that the operator $\kappa_{\mu}$ is an $M$-fuzzy left conucleus. 
By Proposition 6, we have that the mapping $\kappa_{\mu}: L \times M \longrightarrow L$ is an $M$-fuzzy coclosure on $L$. Now, we prove only the condition ( $\left.L \kappa_{4}\right)$. To this end, for $a, b \in L, \beta \in M$ and since $\mu \in M^{L}$ is an $M$-fuzzy left ideal, then $\mu(a \otimes x) \geq \mu(x)$, and therefore,

$$
\begin{aligned}
a \otimes \kappa_{\mu}(b, \beta) & =a \otimes \bigvee\{x \in L: x \leq b, \mu(x) \geq \beta\}, \\
& =\bigvee\{a \otimes x \in L: a \leq b, x \leq b, \mu(x) \geq \beta\}, \\
& =\bigvee\{a \otimes x \in L: a \otimes x \leq a \otimes b, \mu(a \otimes x) \geq \beta\}, \\
& \leq \bigvee\{y \in L: y \leq a \otimes b, \mu(y) \geq \beta\}, \\
& =\kappa_{\mu}(a \otimes b, \beta) .
\end{aligned}
$$

Then, $a \otimes \kappa_{\mu}(b, \beta) \leq \kappa_{\mu}(a \otimes b, \beta)$. The right case follows similarly.

Corollary 3 For $(L, \leq, \otimes),(M, \leq, \odot) \in \mid$ Quant $\mid$ and an $M$-fuzzy ideal $\mu \in M^{L}$ on $L$, the mapping $\kappa_{\mu}: L \times M \longrightarrow L$ defined by the equality,

$\kappa_{\mu}(a, \alpha)=\bigvee\{x \in L: x \leq a, \mu(x) \geq \alpha\} \forall a \in L, \alpha \in M$,

is an $M$-fuzzy ideal conucleus on $L$.

Remark 3 For $a, b \in L$ and $\alpha, \beta \in M$, we have $a, b \leq a \vee b$ and $\alpha \wedge \beta \leq \alpha$, $\beta$, so for an $M$-fuzzy coclosure $\kappa: L \times M \longrightarrow L$, we have that $\kappa(a \vee b, \alpha \wedge \beta) \geq \kappa(a, \alpha), \kappa(b, \beta)$, which implies that $\kappa(a \vee b, \alpha \wedge \beta) \geq \kappa(a, \alpha) \vee \kappa(b, \beta)$.

Proposition 15 For $(L, \leq, \otimes),(M, \leq, \odot) \in \mid$ Quant $\mid$ and an $M$-fuzzy left (resp., right) ideal conucleus $\kappa: L \times M \longrightarrow L$, the mapping $\mu_{\kappa}: L \longrightarrow M$ defined by $\mu_{\kappa}(a)=\bigvee\{\alpha \in$ $M, \kappa(a, \alpha) \geq a, a \in L\}$ is an $M$-fuzzy left (resp., right) ideal of $L$.

Proof Let $\kappa: L \times M \longrightarrow L$ be an $M$-fuzzy left (resp., right) ideal conucleus on $L$. For $a, b \in L$ and $\alpha, \beta \in M$ with $a \leq b$ and $\alpha \geq \beta$, we have

(1) $\mu_{\kappa}(a)=\bigvee\{\alpha \in M: \kappa(a, \alpha) \geq a\}$,

$$
\begin{aligned}
& \geq \bigvee\{\beta \in M: \kappa(b, \beta) \geq b\}, \\
& =\mu_{\kappa}(b) .
\end{aligned}
$$

(2) $\mu_{\kappa}(a) \wedge \mu_{\kappa}(b)=\bigvee\{\alpha \in M: \kappa(a, \alpha) \geq a\} \wedge \bigvee\{\beta \in M: \kappa(b, \beta) \geq b\}$,

$=\bigvee\{\alpha \wedge \beta: \kappa(a, \alpha) \geq a$ and $\kappa(b, \beta) \geq b\}$,

$=\bigvee\{\alpha \wedge \beta: \kappa(a, \alpha) \vee \kappa(b, \beta) \geq a \vee b\}$,

$=\bigvee\{\alpha \wedge \beta: \kappa(a \vee b, \alpha \wedge \beta) \geq \kappa(a, \alpha) \vee \kappa(b, \beta) \geq a \vee b\}$,

$\leq \bigvee\{\alpha \wedge \beta: \kappa(a \vee b, \alpha \wedge \beta) \geq a \vee b\}$

$=\mu_{\kappa}(a \vee b)$.

(3) $\mu_{\kappa}(a \otimes b)=\bigvee\{\beta \in M: \kappa(a \otimes b, \beta) \geq a \otimes b\}$

$=\bigvee\{\beta \in M: a \otimes \kappa(b, \beta) \geq a \otimes b\}$,

$\geq \bigvee\{\beta \in M: \kappa(b, \beta) \geq b\}$

$=\mu_{\kappa}(b)$.

Similarly, $\mu_{\kappa}(a \otimes b) \geq \mu_{\kappa}(a)$.

Corollary 4 For $(L, \leq, \otimes),(M, \leq, \odot) \in \mid$ Quant $\mid$ and an M-fuzzy ideal conucleus $\kappa$ : $L \times M \longrightarrow L$, the mapping $\mu_{\kappa}: L \longrightarrow M$ defined by

$\mu_{\kappa}(a)=\bigvee\{\alpha \in M, \kappa(a, \alpha) \geq a, a \in L\}$ is an M-fuzzy ideal of $L$.

The following lemma provides an important description of $M$-fuzzy left (resp., right) ideal conuclei for a unital quantale in terms of the residuum $\searrow$ (resp., $\swarrow$ ). 
Lemma 4 Let $(L, \leq, \otimes),(M, \leq, \odot) \in \mid$ Quant $\mid$ and $\kappa: L \times M \longrightarrow L$ be an $M$-fuzzy left (resp., right) ideal conucleus on a quantale $L$. Then,

$\kappa(a, \alpha) \searrow \kappa(b, \beta)=\kappa(a, \alpha) \searrow b($ resp.,$\kappa(b, \beta) \swarrow \kappa(a, \alpha)=b \swarrow \kappa(a, \alpha))$,

for all $a, b \in L, \alpha, \beta \in M$.

Proof By (Proposition 1(4)), $a \leq b \Rightarrow c \searrow a \leq c \searrow b$,

$$
\Rightarrow b \searrow c \leq a \searrow c \text {. }
$$

If $\kappa$ is an $M$-fuzzy left ideal conucleus on $L$, then since $\kappa(b, \beta) \leq b$, we have

$\kappa(a, \alpha) \searrow \kappa(b, \beta) \leq \kappa(a, \alpha) \searrow b$, and $b \searrow \kappa(a, \alpha) \leq \kappa(b, \beta) \searrow \kappa(a, \alpha)$.

Thus producing

$$
\kappa(a, \alpha) \searrow \kappa(b, \beta)=\kappa(a, \alpha) \searrow b .
$$

The argument for $\swarrow$ proceeds similarly.

Lemma 5 Let $L$ be a unital quantale. A mapping $\kappa: L \times M \longrightarrow L$ is an $M$-fuzzy left (resp., right) ideal conucleus on $L$ if

$\kappa(a, \alpha) \searrow \kappa(b, \beta)=\kappa(a, \alpha) \searrow b($ resp.,$\kappa(b, \beta) \swarrow \kappa(a, \alpha)=b \swarrow \kappa(a, \alpha))$,

for all $a, b \in L, \alpha, \beta \in M$.

Proof Suppose that $\kappa(b, \beta) \swarrow \kappa(a, \alpha)=b \swarrow \kappa(a, \alpha)$ for all $a, b \in L, \alpha, \beta \in M$, and $e_{L}$ be unit of $L$.

(i) $e_{L} \otimes \kappa(a, \alpha) \leq \kappa(a, \alpha) \Rightarrow e_{L} \leq \kappa(a, \alpha) \swarrow \kappa(a, \alpha)=a \swarrow \kappa(a, \alpha)$

$$
\begin{aligned}
& \Rightarrow e_{L} \otimes \kappa(a, \alpha) \leq a \\
& \Rightarrow \kappa(a, \alpha) \leq a .
\end{aligned}
$$

(ii) If $a \leq b$, and $\beta \leq \alpha$, then

$$
\begin{aligned}
e_{L} \otimes \kappa(a, \alpha) \leq a \leq b & \Rightarrow e_{L} \leq b \swarrow \kappa(a, \alpha)=\kappa(b, \beta) \swarrow \kappa(a, \alpha) \\
& \Rightarrow e_{L} \otimes \kappa(a, \alpha) \leq \kappa(b, \beta) \\
& \Rightarrow \kappa(a, \alpha) \leq \kappa(b, \beta) .
\end{aligned}
$$

That is, $\kappa$ is order preserving.

(iii) Since $\kappa(a, \alpha) \swarrow \kappa(a, \alpha) \leq \kappa(\kappa(a, \alpha), \alpha) \swarrow \kappa(a, \alpha)$, then

$e_{L} \otimes \kappa(a, \alpha) \leq \kappa(a, \alpha) \Rightarrow e_{L} \leq \kappa(\kappa(a, \alpha), \alpha) \swarrow \kappa(a, \alpha)$,

$$
\begin{aligned}
& \Rightarrow e_{L} \otimes \kappa(a, \alpha) \leq \kappa(\kappa(a, \alpha), \alpha), \\
& \Rightarrow \kappa(a, \alpha) \leq \kappa(\kappa(a, \alpha), \alpha) . \\
& \Rightarrow \kappa(a, \alpha)=\kappa(\kappa(a, \alpha), \alpha) .
\end{aligned}
$$

That is, $\kappa$ is idempotent.

By (i), (ii), and (iii), we have that $\kappa$ is an $M$-fuzzy coclosure, and therefore, we have

$$
\begin{aligned}
a \otimes \kappa(b, \beta) \leq \kappa(a \otimes \kappa(b, \beta)) & \Rightarrow a \leq \kappa(a \otimes \kappa(b, \beta)) \swarrow \kappa(b, \beta) \\
& \Rightarrow a \leq \kappa(a \otimes b, \beta) \swarrow \kappa(b, \beta) \\
& \Rightarrow a \otimes \kappa(b, \beta) \leq \kappa(a \otimes b, \beta) .
\end{aligned}
$$

Thus, $a \otimes \kappa(b, \beta) \leq \kappa(a \otimes b, \beta)$. So $\kappa$ is an $M$-fuzzy left ideal conucleus. The right case follows similarly.

As a consequence of the above lemmas, we have the following proposition: 
Proposition 16 Let $L$ be a unital quantale. A map $\kappa: L \times M \longrightarrow L$ is an $M$-fuzzy ideal conucleus if and only if

$$
\begin{aligned}
& \kappa(a, \alpha) \searrow \kappa(b, \beta)=\kappa(a, \alpha) \searrow b \text { and } \kappa(b, \beta) \swarrow \kappa(a, \alpha)=b \swarrow \kappa(a, \alpha), \\
& \text { for all } a, b \in L, \alpha, \beta \in M .
\end{aligned}
$$

\section{Acknowledgements}

The authors thank the anonymous referees and the editors for their valuable comments and suggestions on the improvement of this paper.

\section{Authors' contributions}

All authors jointly worked on the results, and they read and approved the final manuscript.

\section{Funding}

Not applicable.

\section{Availability of data and materials}

Not applicable.

\section{Competing interests}

The authors declare that they have no competing interests.

Received: 4 May 2019 Accepted: 17 July 2019

Published online: 22 August 2019

\section{References}

1. Mulvey, C. J.: \&. Suppl. Rend. Circ. Mat. Palermo Ser. 12, 99-104 (1986)

2. Rodabaugh, S. E.: Relationship of algebraic theories to powerset theories and fuzzy topological theories for lattice-valued mathematics. Int. J. Math. Math. Sci. 71, 2007 (2007). Article ID 43645, http://dx.doi.org/10.1155/2007/ 43645

3. Höhle, U.: Prime elements of non-integral quantales and their applications. Order. 32, 329-346 (2015)

4. El-Saady, K.: Topological representation and quantic separation axioms of semi-quantales. J. Egypt. Math. Soc. 24 568-573 (2016)

5. El-Saady, K.: A non-commutative approach to uniform structures. J. Intell. Fuzzy Syst. 31, 217-225 (2016)

6. Zhang, D.: Sobriety of quantale-valued cotopological spaces. Fuzzy Sets Syst. 350, 1-19 (2018)

7. Demirci, M.: Fuzzy semi-quantales, $(l, m)$-quasi-fuzzy topological spaces and their duality. In: 7th International Joint Conference on Computational Intelligence (IJCCI), pp. 105-111. IEEE press, Lisbon, (2015). https://ieeexplore.ieee. org/document/7533270

8. Kubiak, A. T., Šostak, A. P.: Foundations of the theory of $(L, M)$-fuzzy topological spaces. In: Bodenhofer, U., DeBaets, B., Klement, E. P., Saminger-Platz, S. (eds.) Abstracts of the 30th Linz Seminar on Fuzzy Set Theory, pp. 70-73. Johannes Kepler Universität, Linz, (2009)

9. Höhle, U., Šostak, A. P.: Mathematics of Fuzzy Sets:Logic, Topology and Measure Theory. In: Höhle, U., Rodabaugh, S. E. (eds.), pp. 123-272. Kluwer Academic Publishers, Boston, (1999)

10. Rodabaugh, S. E.: Functorial comparisons of bitopology with topology and the case for redundancy of bitopology in lattice-valued mathematics. Appl. Gen. Topol. 9, 77-108 (2008)

11. Bělohlávek, R.: Fuzzy Relational Systems. Kluwer Academic Publishers, NewYork (2002)

12. Hájek, P.: Metamathematics of Fuzzy Logics. Kluwer Academic Publishers, Dordrecht (1998)

13. Rosenthal, K. I.: Quantales and Their Applications. Longman Scientific and Technical, New York (1990)

14. Rodabaugh, S. E.: Topological and Algebraic Structures in Fuzzy Sets, The Handbook of Recent Developments in the Mathematics of Fuzzy Sets,Trends in Logic. In: Klement, E. P., Rodabaugh, S. E. (eds.), pp. 199-234. Kluwer Academic Publishers, Boston/Dordrecht/London, (2003)

15. Rodabaugh, S. E.: A categorical accommodation of various notions of fuzzy topology. Fuzzy Sets and Systems. 9 , 241-265 (1983)

16. Denniston, J. T., Melton, A., Rodabaugh, S. E.: Formal concept analysis and lattice-valued chu systems. Fuzzy Sets and Systems. 216, 52-90 (2013)

17. Gierz, G., Hofmann, K. H., Keimel, K., et al.: Continuous Lattices and Domains. Cambridge University Press, UK (2003)

18. Erné, M., Koslowski, J., A., M., Strecker, G. E.: A primer on galois connections. Ann. N. Y. Acad. Sci. 704, 103-125 (1993)

19. Solovyov, S.: Lattice-valued topological systems as a framework for lattice-valued formal concept analysis. J. Math. 2013(Article ID 506275), 33 (2013). Article ID 506275, http://dx.doi.org/10.1155/2013/506275

20. Wang, S. Q., Zhao, B.: Ideals of quantales. J. Shaanxi Norm. Univ. Nat. Sci. Ed. 31(4), 7-10 (2003). (in Chinese)

21. Ganter, B., Wille, R.: Formal Concept Analysis: Mathematical Foundations. Springer, New York (1996)

22. Tamura, T.: Examples of direct products of semigroups or groupoids. Am. Math. Soc. 31, 419-422 (1962)

23. Petrich, M.: Categories of Algebraic Systems. Springer, New York (1976)

\section{Publisher's Note}

Springer Nature remains neutral with regard to jurisdictional claims in published maps and institutional affiliations. 